\title{
Summary of Practice Considerations for Percutaneous Coronary Intervention of Left Main Bifurcation Disease
}

Tanveer Rab, ${ }^{1}$ J Dawn Abbott, ${ }^{2}$ Mir Babar Basir, ${ }^{3}$ Azeem Latib,, ${ }^{4}$ Gautam Kumar, ${ }^{1}$ Perwaiz Meraj, ${ }^{5}$ Kevin Croce $^{6}$ and Rajesh Davé7

1. Emory University, Atlanta, GA, USA; 2. Brown University, Providence, RI, USA; 3. Henry Ford Health System, Detroit, MI, USA; 4. Montefiore Medical Center, New York, NY, USA; 5. Northwell Health System, New York, NY, USA; 6. Brigham and Women's Hospital, Boston, MA, USA;

7. Ortenzio Heart Center, Holy Spirit Hospital, Camp Hill, PA, USA

DOI: https://doi.org/10.17925/HI.2020.14.2.69

L eft main bifurcation percutaneous coronary intervention is a challenging subset that requires expertise in techniques that are in constant modification. Imaging is important in lesion preparation and optimising outcomes. The interventionalist needs to be highly skilled in the different techniques, as missteps may lead to stent thrombosis and critical in-stent restenosis. Lesion classification between simple and complex identifies those who would best benefit from a two-stent technique. Current technical approaches and practice considerations are summarised in this manuscript.

\section{Keywords}

Left main, percutaneous coronary intervention, current practice

Disclosures: J Dawn Abbott has received research grants (institutional, no financial compensation) from Abbott Vascular Inc., Astra Zeneca, Sinomed, CSL Behring and Biosensors Research, and is a consultant for Philips. Mir Babar Basir is a consultant for Abbott Vascular, Abiomed, Cardiovascular Systems, Chiesi and Zoll. Azeem Latib is a consultant, and is on the Advisory Board, for Medtronic, Abbott, Boston Scientific and Philips. Perwaiz Meraj is a consultant for Abiomed. Kevin Croce has received grant/research support from Abbott, Takeda, Teleflex and CSI, and has received consulting fees/honoraria from Abbott, BSCl, Biotronik, Philips, Abiomed, CSI, Takeda and Cordis, and is a majo stock shareholder/equity for Dyad Medical. Rajesh Davé is a speaker for Abbot Vascular and is a speaker for, and receives fees from, Proctor. Tanveer Rab and Gautam Kumar have nothing to disclose in relation to this article.

Review Process: Double-blind peer review

Compliance with Ethics: This study involves a review of the literature and did not involve any studies with human or animal subjects performed by any of the authors.

Authorship: All named authors meet the criteria of the International Committee of Medical Journal Editors (ICMJE) for authorship for this manuscript, take responsibility for the integrity of the work as a whole and have given final approval for the version to be published.

Access: This article is freely accessible at touchCARDIO.com (c) Touch Medical Media 2020.

Received: 5 November 2020

Accepted: 17 December 2020

Published Online: 7 January 2021

Citation: Heart International. 2020;14(2):69-72

Corresponding Author: Tanveer Rab, Emory

Decatur Hospital, 2701 N. Decatur Road, Decatur,

GA 30033, USA.E: srab@emory.edu

Support: No funding was received in

the publication of this article.
Technical approaches to left main $(L M)$ revascularisation by percutaneous techniques continue to be in evolution. This manuscript will attempt to summarise the current best practices for LM percutaneous coronary intervention (PCI).

Following a clinical decision for PCl of left main bifurcation (LMB) stenosis, based on angiographic, imaging and functional severity, and supported by current guidelines and publications, the following practice considerations are recommended..$^{1-6}$

\section{Angiographic assessment}

Multiple angiographic views should be utilised including the left anterior oblique (LAO) caudal, shallow LAO caudal, right anterior oblique caudal, anteroposterior caudal and LAO cranial views. Procedural strategy and the best working view should be identified. The Medina Classification describes bifurcation lesions and is the most common terminology used in studies involving strategies for LMB PCI. The SYNTAX score, which describes disease complexity, did not discriminate outcomes in contemporary trials of $\mathrm{PCI}$ compared with coronary bypass for LM disease. ${ }^{7-10}$

\section{Imaging}

Guidance with intravascular ultrasound and optical coherence tomography (OCT) should be considered in all stages of $\mathrm{LMB} P \mathrm{PCl}$ for improved outcomes.11-13 Intracoronary imaging during procedural planning identifies the reference vessel diameter, length, extent, composition of plaque and lesion preparation strategy. ${ }^{1,4}$ Intraprocedural OCT for the LM may be challenging but can assist in the wire crossing of stent struts into the side branch (SB) by choosing the appropriate cell (proximal or distal). ${ }^{15,16}$ Post-procedural imaging is essential to evaluate for malapposition and to optimise the minimal stent area of the LMB and SBS.

\section{Lesion classification}

The key to $L M B P C l$ is preservation of the $S B$, generally the left circumflex, without alteration in the main vessel (MV) stent architecture. Complexity of the LMB anatomy is associated with $\mathrm{PCl}$ outcomes. Lesions can be classified as simple or complex according to a number of variables. The DEFINITION II trial validated these variables as described in the DEFINITION criteria. ${ }^{17-19}$ An SB lesion diameter stenosis $\geq 70 \%$ and/or a lesion length $>10 \mathrm{~mm}$ constituted major criteria. Minor criteria included moderate to severe calcification, thrombus-containing lesion, lesion length $>25 \mathrm{~mm}$, multiple lesions, reference vessel diameter $<2.5 \mathrm{~mm}$ or bifurcation angle $<45$ degrees. The presence of one major and/or two minor criteria qualifies as a complex lesion.

The technical approach to LMB PCI depends on whether a provisional or two-stent strategy is planned, which is predominantly based on classifying LMB as simple or complex. 


\section{Key procedural elements}

LM PCl can be performed with a $6 \mathrm{Fr}$ guiding catheter either by radial or femoral access.

Aggressive lesion preparation of the $\mathrm{MV}$ is required with pre-dilatation of the SB, if the two-stent strategy is planned. This may require coronary atherectomy. ${ }^{20,21}$ With preserved left ventricular function, mechanical circulatory support (MCS) is not typically required. MCS should be considered with depressed left ventricular ejection fraction, the presence of occluded right coronary artery, low cardiac output, the need for atherectomy or decompensated heart failure without an opportunity for medical stabilisation. ${ }^{22}$

- The SB must be wired..$^{23}$ This is important since the left circumflex ( $\mathrm{LCX}$ ) artery is the most common SB and supplies a large amount of myocardium.

- The stent is always sized to the distal vessel, generally the left anterior descending (LAD) artery. ${ }^{24}$ For lesions with a variation of $0.75 \mathrm{~mm}$ or greater in proximal and distal MV diameter, selection of a stent platform with sufficient ability for stent expansion to avoid malapposition is necessary. A 1:1 sizing is recommended using angiography, but it is more precise with intravascular imaging.

- The proximal optimisation technique (POT) is essential with balloon inflation of a short non-compliant (NC) balloon sized to the LM (ideally with intravascular imaging) with a stent length of at least an $8 \mathrm{~mm}$ balloon to be safely placed in LM without overlapping the carina. POT is also recommended after kissing balloon inflation (KBI). ${ }^{25-28}$

- $\mathrm{KBI}$ is absolutely necessary after a two-stent technique. ${ }^{29-32}$

- Intracoronary imaging is needed to optimise post-dilation of the stent using NC balloons. A mean stent area at the carinal confluence of $>8 \mathrm{~mm}^{2}$ is mandated with improved outcomes associated with a mean stent area $>10 \mathrm{~mm}^{2} .13,33-35$

\section{Stenting techniques}

The interventionalist should be familiar with at least four stenting techniques based on lesion complexity. ${ }^{17}$

\section{Simple lesion}

- Provisional stenting (PS): bailout strategies with conversion of PS to a two-stent technique;

- T-stenting and with minimal protrusion (also known as the TAP technique); and

- culotte stenting.

\section{Complex lesion}

- Double kiss crush stenting or culotte stenting.

An upfront dedicated two-stent strategy in complex LMB lesions is recommended over a provisional strategy based on a series of trials, including the recent DEFINITION \| study, and is supported by cardiovascular society guidelines. ${ }^{18,36-39}$

\section{Provisional stenting}

The majority of LMB stenosis are simple lesions, and a single stent approach using a provisional technique is used in $>70 \%$ of cases.

- A single stent is used, generally a crossover from the LAD to the LM and preferably covering the entire LM. This is followed by POT with no $\mathrm{SB}$ dilatation, or $\mathrm{KBI}$. The stent is sized to the distal vessel.

- If intervention is required to the $\mathrm{SB}$, either a fresh wire is used or guidewire exchange occurs. The MV wire is pulled back with careful attention to the guiding catheter that is pulled back to prevent deep intubation, which may result in stent distortion or LM injury. The guidewire is inserted into the SB through the most distal cell (closest to the carina). The jailed SB wire is then pulled back and placed in the MV. Alternatively, a fresh wire can be used with a gentle double curve at the tip, crossing the LM into the LAD with the tip pointing upwards. The tip is then gently pulled back with a downward rotation to enter the SB. In select instances, a dual lumen microcatheter can be used to avoid wiring under a proximal strut and to facilitate $\mathrm{SB}$ rewiring. $\mathrm{POT}, \mathrm{KBI}$ and re-POT is then performed. It is important to pay attention to the position of the POT balloon; the distal marker should be proximal to the neocarina to ensure that the neo-metal carina is not pushed back towards the LCX ostia, causing the re-jailing of the SB ostia.

- An alternative strategy is POT-SB inflation and re-POT (PSP). This optimises the result of PS, maintaining circular geometry, reducing SB ostium strut obstruction and access to the LCX, the risk of SB occlusion and global strut malapposition. ${ }^{40}$

If the result of the SB is inadequate after MV stenting with residual dissection, high-grade stenosis, thrombolysis in myocardial infarction (TIMI) flow $<$ III or an fractional flow reserve (FFR) of $<0.75$ (though not validated in $\mathrm{LM} \mathrm{PCl}$, ischaemic thresholds of FFR $<0.80$ and instantaneous wave-free ratio/resting full-cycle ratio of 0.89 are being used), a second stent can be placed using either a T/TAP or culotte technique after guidewire exchange. ${ }^{41-44}$

Special care should be taken during LM stenting to avoid longitudinal stent deformation. Pulling back of the jailed wire or a partially deflated balloon may deep seat the guiding catheter and damage the stent. Optimal control of the guide catheter with disengagement from the LM is crucial to avoid this complication.

\section{Kissing balloon inflations in provisional stenting}

Routine KBIS in PS is not recommended. . $932,45-47$ When indicated, the $L C X$ is rewired through a distal cell overlying the SB. A short NC balloon is used in the unstented SB to prevent the occurrence of dissection and to avoid oval distortion in the LM. Balloon diameters for the LM and SB are chosen according to Murray's law.48,49 The SB is first inflated to 12 atmospheres, then partly deflated back to 4 atmospheres, with subsequent simultaneous inflation of both balloons at 12 atmospheres with simultaneous deflations. Final KBI is mandatory in two-stent techniques, including a PS strategy that converts to a two-stent technique.

\section{Crossover to a two-stent strategy T-stenting and the TAP technique}

T-stenting or the TAP technique is used to optimise the SB, when the $\mathrm{SB}$ is compromised during PS and the results are suboptimal (occluded vessel, < TIMI-3 flow, SB dissection) after POT is performed. ${ }^{42}$ The SB is recrossed with another wire through a distal cell (closest to the carina) and the jailed SB branch wire is withdrawn. After placement of the SB stent, a 'neocarina' is created by the SB stent struts protruding inside the LM at the level of the carina. The SB take-off angle and the site of strut crossing are major determinants of neocarina length. When the SB has a perpendicular take-off (' $T$ '-shape), minimal SB stent protrusion inside the LM is needed to cover the SB ostium successfully. With acute SB angles ( $Y$ shapes), the SB ostia is longer and oval-shaped. Hence there is wider protrusion of the SB stent inside the LM, resulting in a longer neocarina. It is critical to limit protrusion while implanting the $\mathrm{SB}$ stent to $<2 \mathrm{~mm}$. KBI is the final step of the TAP technique. Deflations should be simultaneous or in rapid sequence with the MV balloon first to avoid crushing of the protruding stents, otherwise the protruded stent in the LM will keep the same position as before KBI. ${ }^{48}$ 


\section{Culotte stenting}

Following PS, POT and distal wire recrossing, SB stent struts are dilated. The second stent is then deployed from the SB into the MV, with the proximal struts just proximal to the edge of the MV stent using a stent diameter based on the SB reference diameter. A second POT is performed followed by a second wire exchange and final KBI. ${ }^{44}$

The main disadvantage of this technique is stent under expansion with three layers of stent at the carina. In a closed-cell design there is a 'napkin-ring' restriction of stent expansion. These factors are independent predictors of stent thrombosis and restenosis., ${ }^{37,45}$

\section{Double kissing crush stenting}

\section{There are 11 procedural steps: ${ }^{37,50,51}$}

1. Short $(<2 \mathrm{~mm})$ protrusion of $S B$ stent into the $L M$, with another balloon positioned from the LM to the LAD.

2. Balloon crush: recent data suggest that a short NC balloon sized to the distal LM with the distal tip at the carina may result in a more complete 'crush'. Intravascular imaging can assist in appropriate balloon sizing, and this balloon can be used later for POT.

3. Rewire the proximal cell (not the distal cell, which is the recommended practice when using PS). This should ideally be performed in the LAO caudal projection to allow operators to see that the wire is away from the carina (OCT can be used to direct the wire through a proximal cell).

4. Alternate balloon inflation of the SB and MV balloon to at least 16 atmospheres, followed by the first $\mathrm{KBI}$.

5. After the first $\mathrm{KBI}$, withdraw the wire and balloon from the SB.

6. Stent the MV, which is sized to the distal reference (i.e. the LAD).
7. Perform the first POT with a short NC balloon sized to the distal LM with the distal tip at the carina (this can be the same NC balloon as in step 2).

8. Rewire the SB from the proximal cell.

9. Alternate balloon inflation of the SB and the MV balloon to at least 16 atmospheres.

10. Perform a second $\mathrm{KBI}$.

11. End the procedure with second and final POT.

Final imaging is a must! !11,34

\section{Left main trifurcations}

Trifurcations are encountered in 10\% of cases and pose technical challenges. In these instances, a single stent strategy is recommended, particularly if the SBs have limited disease. When significant SB disease is present, any two-stent technique might be used according to the specific anatomy; a minor SB (LCX or ramus) is generally identified and treated using a provisional technique. Favourable early and long-term results have been reported, even in true trifurcation lesions that are at high risk of restenosis. ${ }^{52,53}$

\section{Duration of dual antiplatelet therapy}

For the two-stent technique, the duration of dual antiplatelet therapy is extended for $>1$-year, as this results in decreased target lesion failure and thrombotic events compared with therapy continued for less than a year. ${ }^{54}$ Hence, unless the patient has a high bleeding risk, long-term dual antiplatelet therapy should be considered. For the single stent provisional approach, standard guideline-directed therapy should be considered. $\square$
1. Jasti V, Ivan $\mathrm{E}$, Yalamanchili V, et al. Correlations between fractional flow reserve and intravascular ultrasound in patients with an ambiguous left main coronary artery stenosis. Circulation. 2004;110:2831-6

2. Bing R, Yong ASC, Lowe HC. Percutaneous transcatheter assessment of the left main coronary artery: Current status and future directions. JACC: Cardiovasc Interv. 2015;8:1529-39.

3. The Task Force on Myocardial Revascularization of the European Society of Cardiology (ESC) and the European Association for Cardio-Thoracic Surgery (EACTS) Developed Association for Cardio-ion with the special contribion of the European Association of Percutan ESC/EACTS guidelines on myocardial revascu 35:2541-619.

4. Fihn SD, Blankenship JC, Alexander KP, et al. 2014 ACC/AHA/AATS/PCNA/SCAI/STS focused update of the guideline for the diagnosis and management of patients with stable ischemic heart disease: A report of the American College of Cardiology/American Heart Association Task Force on Practice Guidelines, and the American Association for Thoracic Surgery, Preventive Cardiovascular Nurses Association, Society for Cardiovascular Angiography and Interventions, and Society of Thoracic Surgeons. J Am Coll Cardiol. 2014;64:1929-49.

5. Riley RF, Henry TD, Mahmud E, et al. SCAl position statement on optimal percutaneous coronary interventional therapy for complex coronary artery disease. Cathet Cardiovasc Interv. 2020;96:346-62.

6. Burzotta F, Lassen JF, Louvard Y, et al. European Bifurcation Club White Paper on stenting techniques for patients with bifurcated coronary artery lesions. Cathet Cardiovasc Interv. bifurcated coronary

7. Lefèvre T, Girasis C, Lassen JF. Differences between the left main and other bifurcations. Eurolntervention. 2015;11:V106-V10

8. Sianos G, Morel MA, Kappetein AP, et al. The SYNTAX score: An angiographic tool grading the complexity of coronary artery disease. Eurolntervention. 2005;1:219-27.

9. Stone GW, Sabik JF, Serruys PW, et al. Everolimus-eluting stents or bypass surgery for left main coronary artery disease. N Engl J Med. 2016;375:2223-35

10. Mäkikallio $T$, Holm NR, Lindsay M, et al. Percutaneous coronary angioplasty versus coronary artery bypass grafting in treatment of unprotected left main stenosis (NOBLE): a prospective, randomised, open-label, non-inferiority trial. Lancet. 2016:388:2743-52.

11. Zhang J, Gao X, Kan J, et al. Intravascular ultrasound versus angiography-guided drug-eluting stent implantation: The ULTIMATE trial. J Am Coll Cardiol. 2018;72:3126-37.

12. Maehara $A$, Mintz $G$, Serruys $P$, et al. Impact of final minimal stent area by IVUS on 3-year outcome after PCI of left main coronary artery disease: the EXCEL trial. J Am Coll Cardiol. 2017;69(11 Suppl.):963.
13. Maehara A. How to use IVUS to guide LM stenting: Evidence and case examples. 2018. Available at: www.tctmd.com/slide/ how-use-ivus-guide-Im-stenting-evidence-and-case-examples (accessed 11 October 2020)

14. Chen C, Chen C, Yan JT, et al. Analysis of myocardial injury in patients with COVID-19 and association between concomitan cardiovascular diseases and severity of COVID-19. Zhonghua Xin Xue Guan Bing Za Zhi. 2020;48:E008.

15. Longobardo L, Mattesini A, Valente S, Di Mario C. OCT-guided percutaneous coronary intervention in bifurcation lesions. Interv Cardiol (London, England). 2019;14:5-9.

16. Alegría-Barrero $\mathrm{E}$ Foin $\mathrm{N}$, Chan $\mathrm{PH}$, et al. Optical coherence tomography for guidance of distal cell recrossing in bifurcation stenting: choosing the right cell matters. Eurolntervention. 2012;8:205-13.

17. Rab T, Sheiban I, Louvard Y, et al. Current interventions for the left main bifurcation. JACC: Cardiovasc Inter 2017:10:849-65.

18. Zhang J-J, Ye F, Xu K, et al. Multicentre, randomized comparison of two-stent and provisional stenting techniques in patients with complex coronary bifurcation lesions: the DEFINITION trial. Euro Heart J. 2020;41:2523-36.

19. Chen SL, Sheiban I, Xu B, et al. Impact of the complexity of bifurcation lesions treated with drug-eluting stents: the DEFINITION study (Definitions and impact of complex the DEFINITION study (Definitions and impact of complex bifurcation leslons on clinical outcomes after perc
coronary intervention using drug-eluting stents). coronary intervention using drug-eluting
JACC Cardiovasc Interv. 2014:7:1266-76.

20. Garcia-Lara J, Pinar E, Valdesuso R, et al. Percutaneous coronary intervention with rotational atherectomy for severely calcified unprotected left main: Immediate and two-years follow-up results. Cathet Cardiovasc Interv. 2012;80:215-20

21. Yabushita H, Takagi K, Tahara S, et al. Impact of rotational atherectomy on heavily calcified, unprotected left main disease. Circ J. 2014;78:1867-72

22. O'Neill WW, Kleiman NS, Moses J, et al. A prospective, randomized clinical trial of hemodynamic support with Impella 2.5 versus intra-aortic balloon pump in patients undergoing high-risk percutaneous coronary intervention: the PROTECT I study. Circulation. 2012;126:1717-27.

23. Hahn JY, Chun WJ, Kim JH, et al. Predictors and outcomes of side branch occlusion after main vessel stenting in coronary side branch occlusion after main vessel stenting in coronary
bifurcation lesions: results from the COBIS II Registry (coronary bifurcation lesions: results from the COBIS II Registry (coron
blfurcation stenting). J Am Coll Cardiol. 2013;62:1654-9.

blfurcation stenting). J Am Coll Cardiol. 2013;62:1654-9.

coronary intervention for coronary bifurcation disease: 11th consensus document from the European Bifurcation Club. Eurolntervention. 2016;12:38-46.

25. Finet $\mathrm{G}$, Gilard $\mathrm{M}$, Perrenot $\mathrm{B}$, et al. Fractal geometry of arteria coronary bifurcations: a quantitative coronary angiography and intravascular ultrasound analysis. Eurolntervention. 2008;3:490-8.
26. Finet $G$, Derimay $F$, Motreff $P$, et al. Comparative analysis of sequential proximal optimizing technique versus kissing balloon inflation technique in provisional bifurcation stenting: Fractal coronary bifurcation bench test. JACC CardiovasC Interv. 2015;8:1308-17.

27. Darremont O, Leymarie JL, Lefevre T, et al. Technical aspects of the provisional side branch stenting strategy. Eurolntervention. 2015;11(Suppl V):V86-90.

28. Andreasen $L N$. Holm NR, Webber B, Ormiston JA. Critical aspects of balloon position during final proximal optimization technique (POT) in coronary bifurcation stenting. Cathet Cardiovasc Interv. 2020;96:31-9.

29. Niemela M, Kervinen $\mathrm{K}$, Erglis A, et al. Randomized comparison of final kissing balloon dilatation versus no final kissing balloon dilatation in patients with coronary bifurcation lesions treated with main vessel stenting: the Nordic-Baltic Bifurcation Study III. Circulation. 2011;123:79-86

30. Gwon HC, Hahn JY, Koo BK, et al. Final kissing ballooning and long-term clinical outcomes in coronary bifurcation lesions treated with 1-stent technique: results from the COBIS registry. Heart. 2012;98:225-31.

31. Yu CW, Yang JH, Song YB, et al. Long-term clinical outcomes of final kissing ballooning in coronary bifurcation lesions treated with the 1-stent technique: Results from the COBIS II registry (Korean coronary bifurcation stenting registry). JACC Cardiovasc Interv. 2015;8:1297-307.

32. Song YB, Park TK, Hahn JY, et al. Optimal strategy for provisiona side branch intervention in coronary bifurcation lesions: 3 -year outcomes of the SMART-STRATEGY randomized trial. JACC Cardiovasc Interv. 2016;9:517-26.

33. Park SJ, Kim YH, Park DW, et al. Impact of intravascular ultrasound guidance on long-term mortality in stenting for unprotected left main coronary artery stenosis. Circ Cardiovasc Interv. 2009;2:167-77.

34. Park S-J, Ahn J-M, Kang S-J. Unprotected left main percutaneous coronary intervention: Integrated use of fractional flow reserve and intravascular ultrasound. J Am Heart ASSOC. 2012;1. doi.org/10.1161/JAHA.112.004556.

35. Stone GW, Kappetein AP, Sabik JF, et al. Five-year outcomes after $\mathrm{PCl}$ or CABG for left main coronary disease. $N$ Eng/ I Med. 2019:381:1820-30

36. Chen SL, Xu B, Han YL, et al. Clinical outcome after DK crush versus culotte stenting of distal left main bifurcation lesions: The 3-year follow-up results of the DKCRUSH-III study. JACC Cardiovasc Interv. 2015;8:1335-42.

37. Chen $\mathrm{SL}$, XU B, Han YL, et al. Comparison of double kissing crush versus Culotte stenting for unprotected distal left main bifurcation lesions: results from a multicenter randomized, prospective DKCRUSH-III study. J Am Coll Cardiol. 2013;61:1482-8.

38. Chen S-L, Zhang J-J, Han Y, et al. Double kissing crush versus provisional stenting for left main distal bifurcation lesions: The 
DKCRUSH-V randomized trial. J Am Coll Cardiol. 2017;70:2605-17.

39. Neumann F-J, Sousa-Uva M, AhIsson A, et al. 2018 ESC/EACTS guidelines on myocardial revascularization Eur Heart J. 2018:40:87-165.

40. Foin N, Torii R, Mortier P, et al. Kissing balloon or sequentia dilation of the side branch and main vessel for provisional stenting of bifurcations: lessons from micro-computed tomography and computational sm.
Cardiovasc Interv. 2012;5:47-56.

41. Koo BK, Kang HJ, Youn TJ, et al. Physiologic assessment of jailed side branch lesions using fractional flow reserve. J Am Coll Cardiol. 2005;46:633-7.

42. Burzotta F, Dzavik V, Ferenc M, et al. Technical aspects of the T and small protrusion (TAP) technique. Eurolntervention. 2015;11(Suppl. V):V91-5.

43. Ferenc M, Gick M, Comberg T, et al. Culotte stenting vs. TAP stenting for treatment of de-novo coronary bifurcation lesions stenting for treatment of de-novo coronary bifurcation lesions
with the need for side-branch stenting: the Bifurcations Bad Krozingen (BBK) II angiographic trial. Eur Heart J. Krozingen (BBK) II
44. Chevalier B, Glatt B, Royer T, Guyon P. Placement of coronary stents in bifurcation lesions by the "culotte" technique Am J Cardiol. 1998;82:943-9.

45. Murasato $Y$, Finet $\mathrm{G}$, Foin $N$. Final kissing balloon inflation: the whole story. Eurolntervention. 2015;11(Suppl. V):V81-5.

46. Park S-J, Ahn J-M, Park H-S, et al. TCT-234 Is final kissing ballooning mandatory in the treatment of distal left main
bat ballooning mandatory in the treatment of distal left main
disease treated by simple cross over stenting? I Am Coll Cardiol. 2014;64(Suppl. 11):B69

47. Chen S-L, Santoso T, Zhang J-J, et al. Clinical outcome of double kissing crush versus provisional stenting of coronary artery bifurcation lesions: The 5-year follow-up results from a randomized and multicenter DKCRUSH-II study (randomized study on double kissing crush technique versus provisional stenting technique for coronary artery bifurcation lesions). Circ Cardiovasc Interv. 2017;10le004497.

48. Mylotte D, Routledge $\mathrm{H}$, Harb T, et al. Provisional side branch-stenting for coronary bifurcation lesions: evidence of improving procedural and clinical outcomes with contemporary techniques. Cathet Cardiovasc Interv. 2013;82:E437-45.

49. Murray $\mathrm{CD}$. The physiological principle of minimum work: I. The vascular system and the cost of blood volume. Proceedings of the National Academy of Sciences of the United States of America. 1926;12:207-14.

50. Zhang J-J, Chen S-L. Classic crush and DK crush stenting techniques. Eurolntervention. 2015;11:V102-V5.

51. Chen SL, Zhang JJ, Han Y, et al. Double kissing crush versus provisional stenting for left main distal bifurcation lesions: DKCRUSH-V randomized trial. J Am Coll Cardiol.

2017:70:2605-17.

52. Ielasi A, Takagi K, Latib A, et al. Long-term clinical outcomes following drug-eluting stent implantation for unprotected dista trifurcation left main disease: the Milan-New Tokyo (MITO) registry. Cathet Cardiovasc Interv. 2014;83:530-8.

53. Sheiban I, Gerasimou A, Bollati M, et al. Early and long-term results of percutaneous coronary intervention for unprotected left main trifurcation disease. Cathet Cardiovasc Interv. 2009; $73: 25-31$

54. Rhee TM, Park KW, Kim CH, et al. Dual antiplatelet therapy duration determines outcome after 2- but not 1-stent strategy in left main bifurcation percutaneous coronary intervention. in left main bifurcation percutaneous coro 